\title{
LINEAR ASSOCIATIVE ALGEBRAS OF INFINITE ORDER WHOSE ELEMENTS SATISFY FINITE ALGEBRAIC EQUATIONS*
}

BY H. H. CONWELL

1. Introduction. It is the purpose of this paper to investigate linear associative algebras of infinite order, whose elements satisfy finite algebraic equations with coefficients in a field $\Xi$. The definition $\dagger$ of an algebra, and the first three postulates $\ddagger$ assumed, will be the same as those employed by L. E. Dickson for a finite algebra, but in place of Dickson's postulates for a finite basis we shall employ postulates IV and V as follows.

Postulate IV. There exists in $A$ a set of elements $₫$ of such a nature that for every $a \neq 0$ there is determined uniquely a positive integer $n$, a set of distinct elements $u_{1}, u_{2}, \cdots, u_{n}$ of $U$, and $n$ non-zero elements $\xi_{1}, \xi_{2}, \cdots, \xi_{n}$ of $\Xi$ such that $a=\sum_{i=1}^{n} \xi_{i} u_{i}$.

Postulate V. For every element $a$ of $A$ there exists a polynomial function $h(\lambda)$, with coefficients in $\Xi$, such that $h(a)=0$.

2. Idempotent Elements; Semi-Nilpotent Algebras.

ThEOREM 1. Every algebra A contains an idempotent element unless all its elements are nilpotent.

For if $a$ is any non-zero non-nilpotent element of $A$ whose minimum $\|$ equation is $g(\lambda)=0$, of degree $n$, then the finite subalgebra $B=\left(a, a^{2}, a^{3}, \cdots, a^{n}\right)$ of $A$, contains an idempotent element.

THEOREM 2. If an algebra $A$ is not semi-nilpotent, $\uparrow$ but con-

* Presented to the Society, April 9, 1932.

$\dagger$ L. E. Dickson, Algebras and their Arithmetics, University of Chicago Press, pp. 9-11, cited hereafter as Dickson.

$\ddagger$ For the convenience of the reader, references will be made to Dickson, wherever possible, whether or not this constitutes the original source.

$\S$ The set $U$ is not assumed enumerable except in the example of $\S 2$, the subalgebra $M$ of $A$ in Theorem 11, and in Theorem 13 .

\| The equation $g(\lambda)=0$ of lowest degree, with rational coefficients and leading coefficient unity, for which $g(a)=0$, will be called the minimum equation of $a$.

I An algebra $A$ will be called semi-nilpotent if all its elements are nilpotent, and semi-simple if it contains no properly nilpotent elements. 
tains a maximal* semi-nilpotent invariant sub-algebra $K$, then $K$ is unique.

For if $K^{\prime}$ is any other semi-nilpotent invariant sub-algebra of $A$, then $K+K^{\prime}$ is invariant in $A$. Moreover, if $k_{1}$ and $k_{1}^{\prime}$ are any two elements of $K$ and $K^{\prime}$, respectively, then $\left(k_{1}+k_{1}^{\prime}\right)^{\alpha \beta}$ $=\left(k_{\alpha}+k_{1}{ }^{\prime \alpha}\right)^{\beta}=k_{\alpha}{ }^{\beta}=0$, where $k_{\alpha}$ is in $K$ and the indices $\dagger$ of $k_{1}^{\prime}$ and $k_{\alpha}$ are $\alpha$ and $\beta$, respectively. Therefore $K+K^{\prime}$ is a seminilpotent invariant sub-algebra of $A$. But $K$ is maximal, hence $K+K^{\prime}=K$, or $K^{\prime}$ is contained in $K$.

Lemma. Any non-zero element a of an algebra $A$, which is not properly nilpotent in $A$, possesses an inverse with respect to some idempotent element $e$ of $A$, that is, an element $x$ such that $a x=e$.

For otherwise, the sub-algebra $a A$ contains no idempotent element and hence is semi-nilpotent and $a$ is properly nilpotent in $A$, contrary to hypothesis.

TheOREm 3. If $a$ and $b$ are any two properly nilpotent elements of an algebra $A$, then their sum $a+b$ is also properly nilpotent in A.

The contrary assumption necessitates the existence in $A$ of elements $x$ and $e$ such that $(a+b) x=a x+b x=p+q=e$, where $e$ is idempotent and $e p e$ and eqe are zero or properly nilpotent. Therefore, if $\alpha$ is the index of $e p e,(e p e)^{\alpha}=(e-e q e)^{\alpha}=0$, and hence $e=a_{1} q e$, $\left(a_{1}\right.$ in $\left.A\right)$. But $a_{1} q e$ is properly nilpotent in $A$, whereas $e$ is idempotent. This contradiction implies that $a+b$ is properly nilpotent in $A$.

Theorem 4. An algebra $A$ which is not semi-nilpotent contains properly nilpotent elements if and only if it possesses a maximal semi-nilpotent invariant sub-algebra $K$, and then the properly nilpotent elements of $A$ coincide with the non-zero elements of $K$.

The first part of the theorem follows at once from the definition of $K$. To prove the second part, represent the aggregate of properly nilpotent elements in $A$ by $B$. By means of Theorem 3 we can show that $B$ is a semi-nilpotent invariant sub-algebra of $A$, and since it contains all the properly nilpotent elements of $A$ it is maximal and hence is identical with $K$ (Theorem 2).

* Dickson, p. 32.

$\dagger$ If $\alpha$ is an integer such that $a^{\alpha}=0$, but $a^{\alpha-1} \neq 0, \alpha$ is the index of $a$. 
An algebra $A$ may be decomposed into the form

$$
A=I+e R+L e+e A e
$$

in which $e$ is idempotent, $I$ contains all elements $x$ of $A$ such that $e x=x e=0, R=I+e R$ contains all elements $y$ of $A$ such that $y e=0$, and $L=I+L e$ contains all elements $z$ of $A$ such that $e z=0$.

Theorem 5. If $e$ is a principal idempotent element of $A$, every non-zero element of $I, L$, and $R$ in (1) is properly nilpotent.

ProOF. $(L e)^{2}=0=(e R)^{2}$. Since $e L R=L R e=0$, it follows that $L R \leqq I$. If $x$ and $z$ are any two non-zero elements of $I$ and $L e$, respectively, then $(x+z)^{n}=x^{n}+z_{n-1},\left(z_{n-1}\right.$ in Le). Since $x$ is nilpotent with an index $\alpha,(x+z)^{2 \alpha}=z_{\alpha-1}^{2}=0,\left(z_{\alpha-1}\right.$ in $\left.L e\right)$. Thus each element of $L$ is zero or nilpotent. Likewise each element of $R$ is zero or nilpotent.

$A R e=0=R e$, and $e L A=0=e L$, hence $A R \leqq R$ and $L A \leqq L$. Therefore the elements of $R$ and $L$, and hence of their intersection $I$, are properly nilpotent in $A$.

Theorem 6. Every algebra $A$ with a principal idempotent element, but no principal unit, has a semi-nilpotent invariant subalgebra $K$.

This theorem is a direct consequence of Theorems 4 and 5 .

Any semi-simple algebra $A$ of finite order which is not simple is reducible.* The proof of this theorem depends upon the theorem, Any invariant sub-algebra of a semi-simple algebra of finite order possesses a principal unit. The following example exhibits the failure of the latter theorem for infinite algebras, and the subalgebra $B$ illustrates the failure of two other theorems.

(1) Every finite linear associative algebra which is not nilpotent contains a principal idempotent element.*

(2) Every finite algebra with no principal unit has a nilpotent invariant sub-algebra.*

ExAmple. Let $A=\left(e, u_{1}, u_{2}, u_{3}, \cdots\right)$ in which $e u_{i}=u_{i} e=u_{i}$, $e^{2}=e, u_{i}{ }^{2}=u_{i}, u_{i} u_{j}=u_{j} u_{i}=0, i \neq j$. Then $A$ is a semi-simple algebra with the principal unit $e$, and an invariant sub-algebra

* Dickson, pp. 49-53. 
$B=\left(u_{\mathrm{i}}, u_{2}, u_{3}, \cdots\right)$ which contains no principal unit, no principal idempotent element and no nilpotent invariant subalgebra.

3. Difference Algebras.* THEOREM 7. If an algebra $A$ is not semi-nilpotent, but contains the maximal semi-nilpotent invariant sub-algebra $K$, then the difference algebra $A-K$ contains no seminilpotent invariant sub-algebra.

For suppose $A-K$ has a semi-nilpotent invariant sub-algebra $K_{1}$. The elements of $A-K$ are classes [a] of elements of $A$. Represent by $B$ all elements of $A$ belonging to classes [b] of $A-K$ which are in $K_{1}$. From the definition of $K_{1}, B$ is an algebra. If $a$ and $b$ are any two elements of $A$ and $B$, respectively, then $[a][b]=[a b]$ is in $K_{1}$ and also in $B-K$. Hence $B$ is invariant in $A$, and $B-K=K_{1}$, or $B>K$. All elements of $B$ are nilpotent and hence $B$ is a semi-nilpotent invariant sub-algebra of $A$. But $B>K$ contrary to the hypothesis on $K$, therefore $A-K$ contains no semi-nilpotent invariant sub-algebra.

ThEOREM 8. Every idempotent class $[u]$ of $A-K$ contains idempotent elements of $A$.

Proof. $[u]=\left[u^{2}\right]=\left[u^{3}\right]=\cdots=\left[u^{\alpha}\right]$, and $[u] \neq[0]$. Hence $u$ is not nilpotent. If the minimum equation of $u$ is of degree $n$, the finite sub-algebra $B=\left(u, u^{2}, \cdots, u^{n}\right)$, of $A$, contains an idempotent element $e=\xi_{1} u+\xi_{2} u^{2}+\cdots+\xi_{n} u^{n}, \quad\left(\xi_{i}\right.$ in $\left.\boldsymbol{\Xi}\right)$, (Theorem 1). Hence $[e]=\xi[u],\left(\xi=\xi_{1}+\xi_{2}+\cdots+\xi_{n}\right)$. Therefore, $\xi[u]=[e]=[e]^{2}=\xi^{2}[u]^{2}=\xi^{2}[u]$, and hence $\xi=1$, since $\xi=0$ implies $[e]=[0]$ and $e$ nilpotent. Thus $e$ is an idempotent element of $A$, in class $[u]$ of $A-K$.

Theorem 9. If $u$ is a primitive* idempotent element of $A$, and $K$ is a maximal semi-nilpotent invariant sub-algebra of $A$, then $[u]$ is a primitive idempotent element of $A-K$.

ThEOREM 10. If e is a principal* idempotent element of $A$, then $[e]$ is a principal idempotent element of $A-K$ and is identical uith its principal unit.*

Theorem 9 is easily proved by means of Theorems 1 and 8, while Theorem 10 follows readily from Theorems 5 and 6 .

* Dickson, pp. 36-40, 55, 49, 15. 
TheOREM 11. If $A$ has the maximal semi-nilpotent invariant sub-algebra $K$, and $A-K$ contains a simple* matric algebra $M$, with an enumerable base, then $A$ contains a sub-algebra equivalent to $M$.

Proof. Let $M=\left[\epsilon_{i j}\right]$ be a simple matric algebra of classes, in which $\left[\epsilon_{i j}\right]\left[\epsilon_{j k}\right]=\left[\epsilon_{i k}\right],\left[\epsilon_{i j}\right]\left[\epsilon_{h k}\right]=[0],(j \neq h, i, k, j, h=1,2$, $3, \cdots)$. With $e_{11}$ in $\left[\epsilon_{11}\right]$, (Theorem 8 ), as a basis for induction we shall first prove that $A$ contains idempotent elements $e_{11}, e_{22}, \ldots$ whose products in pairs are zero and such that $e_{i i}$ is in $\left[\epsilon_{i i}\right]$.

Let $s=\sum_{i=1}^{n-1} e_{i i}$, where $e_{i i} e_{j j}=0, i \neq j$, and $e_{i i}$ is in $\left[\epsilon_{i i}\right]$. Let $b_{n}$ be any element of $A$ in $\left[\epsilon_{n n}\right]$ and let $a_{n}=b_{n}-s b_{n}-b_{n} s+s b_{n} s$. Then $e_{i i} a_{n}=0=a_{n} e_{i i}$, and $\left[a_{n}\right]=\left[b_{n}\right]=\left[\epsilon_{n n}\right]$, and hence $\left[a_{n}\right]\left[\epsilon_{i i}\right]$ $=[0]=\left[\epsilon_{i i}\right]\left[a_{n}\right],(i=1,2, \cdots, n-1)$. The sub-algebra $B=\left(a_{n}\right.$, $\left.a_{n}^{2}, \cdots, a_{n}^{k}\right)$, of $A$, contains an idempotent element $e_{n n}$ in $\left[\epsilon_{n n}\right]$, (Theorem 8), such that $e_{n n} e_{i i}=0=e_{i i} e_{n n}, i \neq n$. Therefore $A$ contains idempotent elements $e_{11}, e_{22}, e_{33}, \cdots$ whose products in pairs are zero and such that $e_{i i}$ is in $\left[\epsilon_{i i}\right]$ of $M$.

Now consider the non-zero elements $a_{1 j}$ and $b_{j 1}$ of $A$, in [ $\left.\epsilon_{1 j}\right]$ and $\left[\epsilon_{j 1}\right]$ of $M$, respectively. Let $e_{1 j}=e_{11} a_{1 j} e_{j j}$, and $a_{j 1}=e_{j j} b_{11} e_{11}$. Then $\left[e_{1 j}\right]\left[a_{j 1}\right]=\left[\epsilon_{11}\right]$ and hence $e_{1 j} a_{j 1}=e_{11}+k,(k$ in $K$, with in$\operatorname{dex} \alpha)$. Moreover, $e_{11} \epsilon_{1 j} a_{j 1}=e_{1 j} a_{j 1}$, and hence $e_{11}\left(e_{11}+k\right)=e_{11}+k$, or $e_{11} k=k$. Similarly, $k e_{11}=k$. Let $e_{j 1}=a_{j 1}-a_{j 1} k+a_{j} k^{2}-a_{j} k^{3}$ $+\cdots+(-1)^{\alpha-1} a_{j 1} k^{\alpha-1}$. Then

$$
\begin{array}{r}
e_{1 j} e_{j 1}=\left(e_{11}+k\right)-\left(k+k^{2}\right)+\cdots+(-1)^{\alpha-1}\left(k^{\alpha-1}+k^{\alpha}\right) \\
=e_{11}+k^{\alpha}=e_{11} .
\end{array}
$$

Similarly, $e_{j 1} e_{1 j}=e_{j j}+k_{1},\left(k_{1}\right.$ in $\left.K\right)$. Hence from the definitions of $a_{j 1}, e_{1 j}$, and $e_{j 1}$, it follows that $e_{j j} k_{1}=k_{1} e_{j j}=k_{1}$. Therefore $\left(e_{j j}+k_{1}\right)^{2}=e_{j j}+2 k_{1}+k_{1}^{2}=\left(e_{j i} e_{1 j}\right)^{2}=e_{j j}+k_{1}$, and $k_{1}^{2}=-k_{1}$, from which $k_{1}^{2 \alpha}=-k_{1}=0$, since $k_{1}$ is nilpotent. Therefore, $e_{j 1} e_{1 j}=e_{j j}$.

Let $e_{i j}=e_{i 1} e_{1 j},\left(e_{i 1}\right.$ in $\left[\epsilon_{i 1}\right], e_{1 j}$ in $\left.\left[\epsilon_{1 j}\right]\right)$. Then $e_{i j}$ is in $\left[\epsilon_{i j}\right]$. From the definitions of $e_{i j}$ and $e_{1 j}$, and preceding relations, it follows that

* If $A$ is an algebra of matrices having only a finite number of non-zero elements in each matrix, and if the base elements $\left(u_{k}\right),(k, h=1,2, \cdots)$, of $A$ are matrices whose elements are all zero except that in the $k$ th row and $h$ th column, which is unity, then the algebra $M=\left(\epsilon_{i j}\right)$ equivalent to $A$, in which $(i, j)$ have the same range as $(k, h)$, will be called a simple matric algebra with the base elements $\epsilon_{i j}$. Thus $\epsilon_{i j} \epsilon_{j k}=\epsilon_{i k}, \epsilon_{i j} \epsilon_{h k}=0, h \neq j$. 
$e_{i j} e_{j h}=e_{i k}, \quad$ and $\quad e_{i j} e_{h k}=\left(\epsilon_{i 1} e_{1 j} e_{j j}\right)\left(e_{h h} \epsilon_{h 1} e_{1 k}\right)=0, \quad j \neq h$.

This completes the proof that $A$ contains elements $e_{i j}$ in classes $\left[\epsilon_{i j}\right]$ of $M$, respectively, and such that the elements $e_{i j}$ constitute the base of a sub-algebra of $A$ which is equivalent to $M$.

4. Canonical Form of the Matrix $R$. Every linear associative algebra satisfying the postulates of this paper is equivalent* to a matric algebra, and hence an element of the algebra, and its matric representation, must satisfy the same minimum equation. Every finite matrix can be reduced to a rational canonical $\dagger$ form, but this is not true for matrices of infinite order.

Let $R_{a}=\left(\rho_{k j}\right)$ correspond to $a=\sum_{i} \xi_{i} u_{i}$, where $\rho_{k j}=\sum_{i} \xi_{i} \gamma_{i j k}$, and $\gamma_{i j k}$ is the coefficient of $u_{k}$ in the product $u_{i} u_{j}$. Since the number of terms $u_{k}$ in any product $u_{i} u_{j}$ is finite, $R_{a}$ will have but a finite number of non-zero elements in any column. Let $W_{i}=\sum_{k} \rho_{k i} u_{i}$, where $k$ has an infinite range, but only a finite number of the $\rho_{k i}$ are not zero. Let $x_{11}=\sum_{i} c_{i} u_{i}$, and $X_{11}=\sum_{i} c_{i} W_{i}$, be any two corresponding finite linear functions of $u_{i}$ and $W_{i}$, respectively, $c_{i}$ in $\Xi$. Then $X_{11}=\sum_{i k} c_{i} \rho_{k i} u_{k}=x_{12}$, and similarly, $X_{12}=\sum_{i k} c_{i} \rho_{k i} W_{k}=\sum_{i k h} c_{i} \rho_{k i} \rho_{h k} u_{h}=\sum_{i h} c_{i} \rho_{h i}^{\prime} u_{h}=x_{13}, \quad X_{13}=$ $\sum_{i h} c_{i} \rho_{h i}^{\prime} W_{h}=\sum_{i j} c_{i} \rho_{j i}^{(2)} u_{j}=x_{14}$, etc., where $\rho_{k j}^{(r-1)}$ is the element in the $k$ th row and $j$ th column of $\left(\rho_{k j}\right)^{r}$. These relations may be written more briefly in the form

$$
\begin{gathered}
x_{11}=\sum_{i} c_{i} u_{i}, X_{11}=\sum_{i j} c_{i} \rho_{j i} u_{j}=x_{12}, X_{12}=\sum_{i j} c_{i} \rho_{j i}^{\prime} u_{j}=x_{13}, \\
\cdot \cdot, X_{1 r}=\sum_{i j} c_{i} \rho_{j i}^{(r-1)} u_{j}=x_{1 r+1}, \cdot \cdot \cdot \cdot
\end{gathered}
$$

If the minimum equation of $a$ is $\lambda^{m}+\xi_{m} \lambda^{m-1}+\cdots+\xi_{1} \lambda+\xi_{0}$ $=0$, then

$$
\begin{aligned}
& \rho_{j i}^{(m-1)}+\xi_{m} \rho_{j i}^{(m-2)}+\cdots+\xi_{1} \rho_{j i}=0,(i \neq j, i, j=1,2,3, \cdots), \\
& \rho_{i i}^{(m-1)}+\xi_{m} \rho_{i i}^{(m-2)}+\cdots+\xi_{1} \rho_{i i}+\xi_{0}=0 .
\end{aligned}
$$

Therefore

$$
X_{1 m}+\xi_{m} X_{1 m-1}+\cdots+\xi_{1} X_{11}+\xi_{0} x_{11}=0,
$$

* M. H. Ingraham, this Bulletin, vol. 32 (1926), p. 589.

$\dagger$ L. E. Dickson, Modern Algebraic Theories, p. 89. 
which proves the following theorem.

THEOREM 12. If $\left(\rho_{k j}\right)$ satisfies an equation of degree $m$, with rational coefficients, the number of linearly independent linear functions $x_{11}, X_{11}, \cdots, X_{1 i-1}$, as determined in (1), is $\alpha \leqq m$.

In what follows we shall write (1) in the form

$$
\begin{aligned}
& X_{11}=x_{12}, \quad X_{12}=x_{13}, \cdots, \quad X_{1 \alpha-1}=x_{1 \alpha}, \\
& X_{1 \alpha}=\sum_{i=1}^{\alpha} \xi_{1 i} x_{1 i}=\left[x_{11}, \cdots, x_{1 \alpha}\right] .
\end{aligned}
$$

Any finite linear expression $\sum_{i} c_{i} u_{i}$ leads to a chain of the type of $\left(1^{\prime}\right)$. The maximal length of all such chains is $\alpha_{1} \leqq m$.

THEOREM 13. If $R_{a}=\left(\rho_{k j}\right)$ corresponds to the element $a=\sum_{i} c_{i} u_{i}$ of a rational linear associative algebra $A$, with the enumerable base $\left(u_{1}, u_{2}, u_{3}, \cdots\right)$, then $R_{a}$ may be reduced to a canonical form defined by

$$
\begin{aligned}
X_{i_{i 1}} & =x_{i_{i} 2}, \cdots, \quad X_{i_{i \alpha_{i-1}}}=x_{i_{i} \alpha_{i}}, \\
X_{i_{i} \alpha_{i}} & =\left[x_{i_{i 1}}, x_{j_{i} 2}, \cdots, x_{j_{i} \alpha_{i}}\right] \\
(i & \left.=1,2, \cdots, p \leqq \alpha_{1} ; j_{i}=1,2,3, \cdots\right),
\end{aligned}
$$

in which $X_{j_{i}}, X_{j_{i}}, \cdots, X_{j_{i} \alpha_{i}}$ are the elements of the $j_{i}$ th chain of length $\alpha_{i}$, each of which is linearly independent of all preceding chains of length $\alpha_{1}, \alpha_{2}, \cdots, \alpha_{i-1}$ and the preceding elements of the $j_{i}$ th chain of length $\alpha_{i}$. Moreover, $\alpha_{1}$ is the maximal length of all possible chains, and in general, $\alpha_{i}$ is the maximal length of all chains of length less than $\alpha_{i-1}$ which are linearly independent of all chains of length $\alpha_{1}, \alpha_{2}, \cdots, \alpha_{i-1}$.

Proofs of this theorem for the finite case that have been given by L. E. Dickson (Modern Algebraic Theories, p. 90), or M. H. Ingraham (this Bulletin, vol. 39, p. 379), may be extended to the infinite case, provided we secure the leaders of successive chains as follows.

The leader, $x_{11}=\sum_{i} c_{i} u_{i}$, of the first chain of length $\alpha_{1}$ may be obtained from successive trials of the linear functions $\sum_{i} c_{i} u_{i}$ such that $\sum i=n,\left(n=1,2, \cdots, k_{1}\right)$. The leaders of the succeeding chains of length $\alpha_{1}$ may be obtained in the same way with $\sum i=n,\left(n=k_{1}, k_{1}+1, k_{1}+2, \cdots, k_{2} ; k_{2}, k_{2}+1, \cdots, k_{3}\right.$; etc.). To obtain the chains of length $\alpha_{2}, \alpha_{3}$, etc., repeat the entire 
process for $\alpha_{2}$, then for $\alpha_{3}$, etc. With $\left(1^{\prime}\right)$ as a basis for induction we can prove that the $k$ th chain of length $\alpha_{1}$ can be expressed in the form

(3) $X_{k 1}=x_{k 2}, \cdots, X_{k \alpha_{1}-1}=x_{k \alpha_{1}}, X_{k \alpha_{1}}=\left[x_{k 1}, x_{k 2}, \cdots, x_{k \alpha_{1}}\right]$.

Similarly, all chains of length $\alpha_{i}$ can be reduced to forms of the type of $(3)$. We can thus reduce $\left(\rho_{k j}\right)$ to the canonical form defined by (2), in which there are infinitely many partial transformations, but not more than $\alpha_{1}$ different lengths to the chains.

Each of the base elements $u_{1}, u_{2}, u_{3}, \cdots$, either is itself an element of (2), or else is a finite linear combination of such elements. For, in the process of determining the complete canonical transformation, any $u_{i}$ which is finitely linearly independent of all previously determined $x_{j k}$ is taken as the leader of a new chain and is therefore itself an $x_{j k}$. Hence it is possible to determine uniquely each of the variables $u_{i}$ in terms of the variables $x_{j k}$.

The characteristic determinant of the matrix of any chain is divisible by that of any other chain of equal or lower order. Hence the minimum function of $\left(\rho_{k j}\right)$ is identical with that of the chain of maximal length, and if $\left(\rho_{k j}\right)$ satisfies no finite equation it cannot be reduced to a rational canonical form with a chain of maximal length $\alpha$.

Beloit College 